\title{
Autonomous Replication and Addition of Telomerelike Sequences to DNA Microinjected into Paramecium tetraurelia Macronuclei
}

\author{
DAVID GILLEY, ${ }^{1}$ JOHN R. PREER, JR., ${ }^{1}$ KARL J. AUFDERHEIDE, ${ }^{2}$ AND BARRY POLISKY ${ }^{1 *}$ \\ Program in Molecular, Cellular and Developmental Biology, Department of Biology, Indiana University, \\ Bloomington, Indiana 47405, ${ }^{1}$ and Department of Biology, Texas A\&M University, \\ College Station, Texas 77843-3258
}

Received 6 May 1988/Accepted 1 August 1988

\begin{abstract}
Paramecium tetraurelia can be transformed by microinjection of cloned serotype A gene sequences into the macronucleus. Transformants are detected by their ability to express serotype $A$ surface antigen from the injected templates. After injection, the DNA is converted from a supercoiled form to a linear form by cleavage at nonrandom sites. The linear form appears to replicate autonomously as a unit-length molecule and is present in transformants at high copy number. The injected DNA is further processed by the addition of parameciumtype telomeric sequences to the termini of the linear DNA. To examine the fate of injected linear DNA molecules, plasmid pSA14SB DNA containing the A gene was cleaved into two linear pieces, a 14-kilobase (kb) piece containing the $A$ gene and flanking sequences and a $2.2-\mathrm{kb}$ piece consisting of the procaryotic vector. In transformants expressing the A gene, we observed that two linear DNA species were present which correspond to the two species injected. Both species had Paramecium telomerelike sequences added to their termini. For the 2.2-kb DNA, we show that the site of addition of the telomerelike sequences is directly at one terminus and within one nucleotide of the other terminus. These results indicate that injected procaryotic DNA is capable of autonomous replication in Paramecium macronuclei and that telomeric addition in the macronucleus does not require specific recognition sequences.
\end{abstract}

Foreign DNA introduced into eucaryotic cells can suffer a variety of fates depending on the sequences introduced and the system used. In mouse $\mathrm{L}$ cells in culture, injection of multiple copies of either supercoiled or linear DNA molecules into nuclei can result in integration into host chromosomes at a limited number of sites of head-to-tail concatamers of the input DNA $(7,18)$. These concatamers result from highly efficient homologous recombination mediated by host recombinational machinery. Alternatively, microinjection of a variety of different supercoiled DNA species into unfertilized Xenopus laevis eggs leads to autonomous replication of the injected DNA as supercoiled forms, apparently regardless of sequence $(13,16)$. In the yeast Saccharomyces cerevisiae, foreign supercoiled DNA introduced by transformation replicates autonomously only if it contains distinctive sequences called autonomously replicating sequences (21), presumably reflecting specific recognition of certain sequences by host replication machinery.

We describe here another fate for DNA introduced into the ciliated protozoan Paramecium tetraurelia. We have previously described a transformation system in which exogenous DNA is introduced into the macronuclei of recipients by microinjection (10). Our assay for transformation involves the synthesis of specific cell surface antigens called immobilization antigens (for a review, see reference 19). Paramecia can alter their surface antigens under different environmental conditions. In general, they express only one type at a time. Eleven different serotypes have been described for stock 51 of $P$. tetraurelia, and several of the genes encoding these antigens have been cloned (9). In addition, deletion mutants that lack the serotype $A$ gene have been isolated and characterized (5). Transformants capable of producing functional serotype A surface antigen can be isolated with high efficiency after microinjection of

\footnotetext{
* Corresponding author.
}

cloned serotype A gene-containing DNA into the macronucleus of the $d 12$ deletion mutant (10).

We have shown that the DNA introduced into $P$. tetraurelia is capable of apparent autonomous replication and is maintained at high copy number $(50,000$ to 100,000 copies per cell) until autogamy, when the macronucleus breaks down. The majority of the replicating DNA molecules appeared to be unit-length linear form despite the fact that the input was supercoiled. Here we provide additional evidence for this observation and show that sequences characteristic of Paramecium telomeres are added to the termini of DNA after injection. We demonstrate that injected linear DNA is capable of replicating as a linear form even though it contains sequences derived entirely from a procaryotic source. Our results suggest that the telomere addition system in Paramecium macronuclei does not require preexisting telomeres on the termini of linear molecules to add telomeres.

\section{MATERIALS AND METHODS}

Paramecia. Wild-type cells were $P$. tetaurelia, stock 51.s. The Mendelian $d 12$ mutant was isolated after X-irradiation by J. Forney and L. Epstein (personal communication) as a line unable to express serotype A surface antigen. The $d 12$ line was deleted for the A gene (J. Forney and L. Epstein, unpublished data). Paramecia were cultured either in $\mathrm{Ce}$ rophyl (Cerophyl Co., Kansas City, Mo.) or in baked lettuce medium inoculated with Klebsiella pneumoniae.

Cloned DNA. Plasmid pSA14SB (Fig. 1) has been described previously (10). It consists of a 14-kilobase (kb) fragment of Paramecium DNA containing the A serotype gene and flanking sequences cloned into the 2.2-kb expression vector pT7/T3-18 (Bethesda Research Laboratories, Inc., Gaithersburg, Md.).

Microinjection. Microinjection into the macronuclei of paramecia was done as previously described (10). About $5 \mathrm{pl}$ 


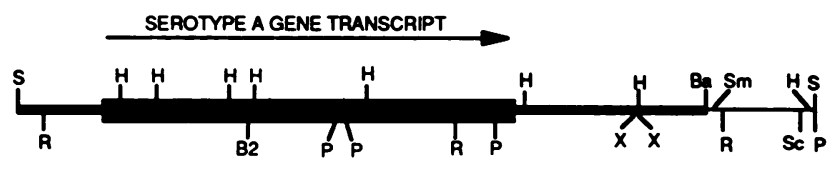

$1 \mathrm{~kb}$

FIG. 1. Schematic diagram of pSA14SB DNA linearized at its unique $S a l$ I site. The construction of this 16.2 -kb plasmid has been described previously (10). It contains the A serotype coding region (C) and flanking Paramecium sequences (-). The arrow denotes the direction of transcription. The vector sequence $(-)$ is pT7/T3-18, consisting of a pUC-type plasmid replication origin and the bla gene conferring ampicillin resistance. The location of several

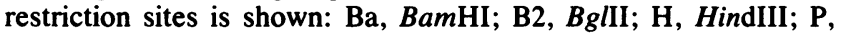
PstI; R, EcoRI; S, SalI; Sc, ScaI; Sm, SmaI; X, XbaI.

of a DNA solution at $3 \mathrm{mg} / \mathrm{ml}$ was injected. For the $16.2-\mathrm{kb}$ plasmid pSA14SB, this corresponds to about 170,000 molecules per macronucleus.

Electrophoresis. Preparation of lysates, purification of DNA, and field inversion gel electrophoresis (FIGE) with 0.8 or $1 \%$ agarose gels were carried out exactly as previously described (10).

Hybridization. Oligonucleotides were produced on an Applied Biosystems DNA synthesizer and labeled at their 5' termini with $\left[\alpha-{ }^{32} \mathrm{P}\right]$ ATP by using T4 polynucleotide kinase under conditions recommended by the enzyme supplier (Bethesda Research Laboratories). Unincorporated counts were removed by passage over a Sephadex G-50 column. Optimal hybridization conditions were determined empirically to be at $50^{\circ} \mathrm{C}$ in $5 \times \mathrm{SSC}(1 \times \mathrm{SSC}$ is $0.15 \mathrm{M} \mathrm{NaCl}$ plus $0.015 \mathrm{M}$ sodium citrate)-20 mM sodium phosphate ( $\mathrm{pH} 7.0$ )$10 \times$ Denhardt solution (15)-7\% sodium dodecyl sulfate (SDS)-100 $\mu \mathrm{g}$ of denatured salmon sperm DNA per ml-10\% dextran sulfate. Filters were washed first at $55^{\circ} \mathrm{C}$ for $1 \mathrm{~h}$ in $\therefore \times$ SSC- $10 \mathrm{mM}$ sodium phosphate (pH 7.0)-10 $\times$ Denhardt sointion-5\% SDS and then washed for $1 \mathrm{~h}$ at $55^{\circ} \mathrm{C}$ in $1 \times$ SSC-1\% SDS.

Nick-translated DNA probes were labeled with $\left[{ }^{32} \mathrm{P}\right] \mathrm{dCTP}$ and hybridized exactly as previously described (10). Filters were washed twice with $2 \times \mathrm{SSC}-0.1 \%$ SDS at $68^{\circ} \mathrm{C}$ for 30 min and then washed once in $0.1 \times \mathrm{SSC}-0.1 \%$ SDS for 30 $\min$ at $68^{\circ} \mathrm{C}$.

Bal 31 nuclease digestion. Bal 31 nuclease was obtained from Bethesda Research Laboratories. DNA was digested with $0.05 \mathrm{U}$ of $\mathrm{Bal} 31$ nuclease for various times. Digestion was carried out at $30^{\circ} \mathrm{C}$ in a volume of $10 \mu$ l containing 200 $\mathrm{mM} \mathrm{NaCl}, 12.5 \mathrm{mM} \mathrm{CaCl}_{2}, 12.5 \mathrm{mM} \mathrm{MgSO}_{4}, 20 \mathrm{mM}$ Tris hydrochloride ( $\mathrm{pH} \mathrm{8.1)}$, and $1 \mathrm{mM}$ EDTA. Reactions were terminated by adding EDTA to a final concentration of 20 $\mathrm{mM}$ and quick freezing. Electrophoresis dyes were added, and samples were electrophoresed on a $1 \%$ agarose gel in Tris-acetate buffer.

\section{RESULTS}

Sensitivity of microinjected DNA to $\mathrm{Bal} 31$ nuclease treasment. We have previously shown that microinjected supercoiled plasmid pSA14SB DNA containing the serotype A surface antigen was converted to a species that comigrated with linear plasmid DNA when total DNA from $d 12$ paramecium transformants was analyzed by FIGE (10). Restriction analysis of these molecules indicated that their termini were heterogeneous with regard to sequence, indicating that the apparent cleavage of the supercoiled form did not occur

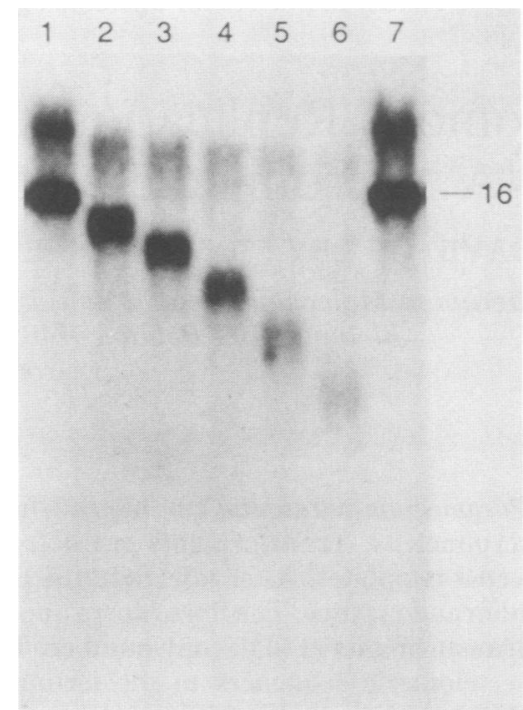

FIG. 2. Bal 31 nuclease analysis of DNA after injection. DNA was prepared from $d 12$ cells 20 fissions after injection of a single cell with supercoiled pSA14SB DNA. The cell line expressed the injected A serotype gene. DNA from the transformant was treated with $0.25 \mathrm{U}$ of $\mathrm{Bal} 31$ nuclease per $\mathrm{ml}$. Samples $(0.4 \mu \mathrm{g}$ each) were removed at $0,10,20,30,40$, and $50 \mathrm{~min}$ after $\mathrm{Bal} 31$ nuclease addition (lanes 1 through 6) and electrophoresed on a $1 \%$ agarose gel. The DNA was transferred to nitrocellulose and probed with labeled pSA14SB DNA. The autoradiogram was exposed for $23 \mathrm{~h}$. The DNA loaded in lane 7 was identical to that loaded in lane 1 . The position of linear pSA14SB mobility in the gel is noted $(16 \mathrm{~kb})$.

at a small number of specific sites. To obtain further evidence for a linear conformation, we investigated the susceptibility of the A gene-hybridizable material in transformants to Bal 31 nuclease treatment. Purified total transformant DNA was treated with $\mathrm{Bal} 31$ nuclease for various times and analyzed on an agarose gel. The DNA was transferred to nitrocellulose and probed with nick-translated ${ }^{32} \mathrm{P}$-labeled pSA14SB DNA.

The results show that the major hybridizable component is progressively reduced in size by $\mathrm{Bal} 31$ nuclease (Fig. 2, lanes 2 through 6). A minor component that electrophoreses more slowly is also affected by $\mathrm{Bal} 31$ nuclease. The molecular nature of this minor component is not known. In control experiments under identical conditions, we showed that pSA14SB DNA linearized at its unique KpnI site, Bal 31 nuclease treated, and then cleaved into six fragments with HindIII showed progressive reduction in size from the expected KpnI termini, consistent with the conclusion that Bal 31 nuclease was functioning as expected (data not shown). Thus, by two criteria, viz., mobility of the intact species on FIGE gels and susceptibility to $\mathrm{Bal} 31$ nuclease, it appears that the majority of supercoiled DNA microinjected into macronuclei is converted to linear form.

Presence of telomerelike sequences in DNA after microinjection. Given that the major transforming DNA species was linear and present in high copy number as a consequence of autonomous replication, an obvious problem concerned the molecular nature of its termini. This problem follows from the fact that all known DNA polymerases require an RNA or DNA primer with a $3^{\prime} \mathrm{OH}$ group to prime DNA synthesis in a 5'-to-3' direction (14). Replication of a linear molecule would leave a $5^{\prime}$ gap at each end after removal of an RNA primer. As an initial approach to this question, we investigated whether sequences characteristic of Paramecium tel- 


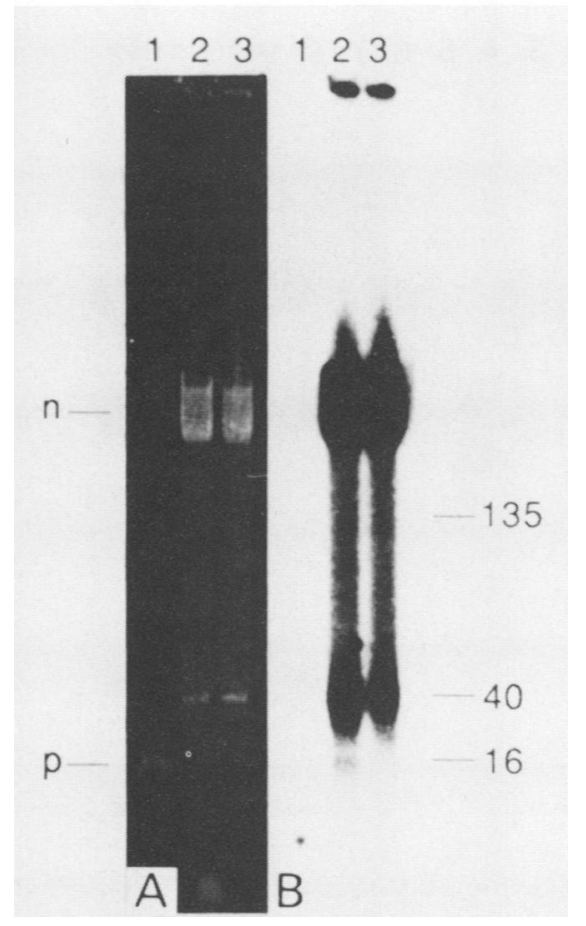

FIG. 3. Addition of telomerelike sequences to injected DNA. Total DNA from a transformant line originally injected with supercoiled pSA14SB DNA was electrophoresed on a $1 \%$ agarose FIGE gel. The DNA was transferred to nitrocellulose and hybridized with ${ }^{3}$ P-labeled synthetic 24-nucleotide oligomer complementary to Paramecium telomeres. Lane $1,0.5 \mu \mathrm{g}$ of pSA14SB DNA linearized with SalI, which serves as marker for the injected DNA; lane 2, DNA from the $d 12$ transformant; lane 3, DNA from untransformed d12 paramecia. (A) Ethidium bromide staining pattern; (B) autoradiogram (72-h exposure). $n$, Bulk macronuclear chromosomal DNA; $p$, position of the linear plasmid. Numbers on the right are size markers in kilobases. As noted in the text, the strong hybridization signal at approximately $40 \mathrm{~kb}(\mathrm{~B}$, lanes 2 and 3$)$ is not mitochondrial DNA.

omeres were added to the injected DNA. Paramecium telomeres consist of a random mixture of $C_{4} A_{2}$ and $C_{3} A_{3}$ repeats (8). Transformant DNA was electrophoresed by the FIGE procedure, which separates macronuclear DNA and linear mitochondrial DNA from the injected DNA species (10). The DNA was transferred to nitrocellulose and probed with a labeled 24-nucleotide synthetic oligonucleotide of the sequence $\left(C_{4} A_{2}\right)_{2}\left(C_{3} A_{3}\right)_{2}$, which is complementary to one strand of the Paramecium telomere sequence. The results of the blot hybridization are shown in Fig. 3B. The ethidium bromide-stained gel is shown in Fig. 3A. Figure 3B shows that the telomeric probe hybridized strongly to macronuclear DNA, which consists of a heterogenous collection of linear molecules ranging in size from about 150 to $400 \mathrm{~kb}(10,20)$. Also, a strong signal is visible at the mobility of mitochondrial DNA, which was an unanticipated result. Further experiments with purified mitochondrial DNA have indicated that it does not hybridize with the telomere probe ( $\mathrm{J}$. Greenlee, unpublished data), indicating that the telomere signal in the FIGE gel blot is due to a macronuclear DNA species of approximately $40 \mathrm{~kb}$ which comigrates with mitochondrial DNA. Ribosomal DNA genes of $P$. tetraurelia, known to exist as a heterogeneous collection of circular and linear molecules of 40 to $50 \mathrm{~kb}(6)$, are present in the $40-\mathrm{kb}$ region of FIGE gels (Greenlee, unpublished data) and pos- sibly are responsible for the 40-kb telomere-hybridizing signal. In addition to this signal, in transformant DNA samples but not in control DNA, hybridization of the telomere probe is evident at the approximate mobility of the linear pSA14SB species (Fig. 3B, lane 2). Note that under these hybridization conditions, the telomere probe does not hybridize with purified linear pSA14SB DNA (Fig. 3A and $B$, lanes 1 ), which was present at a 100 -fold excess over the amount present in the transformant DNA. We conclude that the injected DNA has acquired Paramecium telomerelike sequences, presumably at its termini.

Transformation with linear DNA species. We have shown previously that injected supercoiled molecules are cut at different positions to generate linear species (10). This result precluded a simple assessment of the termini present in the population of replicating molecules. Introduction of linearized DNA potentially permits assessment of the fate of defined termini. Accordingly, we injected pSA14SB DNA that had been cleaved with BamHI and SalI endonucleases. Cleavage with these enzymes produced two fragments, one of $14 \mathrm{~kb}$, consisting entirely of Paramecium sequences including the serotype A gene and flanking sequences, and the other of $2.2 \mathrm{~kb}$, comprising the procaryotic plasmid vector pT7/T3-18 (Fig. 1). The latter DNA contains a pUCtype plasmid replication origin and the $\beta$-lactamase gene.

We observed that the transformation efficiency with cleaved plasmid was about $20 \%$, compared with about $40 \%$ for supercoiled DNA (efficiency was defined as the percentage of $d 12$ mutant $P$. tetraurelia injected that become serotype $A$ after 10 fissions). When transformant DNA was fractionated by FIGE, blotted, and probed with labeled pSA14SB DNA, a heterogeneous hybridization signal was detected in the 12- to $15-\mathrm{kb}$ region of the gel (Fig. 4, lane 1). The majority of the hybridizing material was smaller than the input linear DNA. Note that the injected 14-kb linear fragment was converted to a wider range of sizes than injected supercoiled DNA was (Fig. 4, lane 3). The intensity of the signal indicated that the approximate copy number of the 12 to $15-k b$ DNA was about 50,000 to 100,000 per cell, i.e., in the same range as that displayed by transformants injected with supercoiled DNA (10).

When transformant DNA was subjected to restriction analysis with enzymes that cut once within the 14-kb fragment and probed with labeled pSA14SB DNA, the two fragments of molecular size expected for the intact, input linear fragment were visible on DNA blots (Fig. 5). A heterogeneous hybridization signal was also evident in these lanes, indicating that a substantial fraction of input molecules were reduced in size. Given the heterogeneity of the uncut material, it is obvious that some of the expected fragments generated by restriction cleavages could arise from separate sublinear molecules rather than from intact 14-kb molecules. Thus, although it is difficult to estimate the amount of intact 14-kb linear molecules in the transformants, it is evident that a fraction of the molecules contains restriction fragments of the expected sizes. These results indicate that the injected 14-kb fragment undergoes some degradation during or after injection to generate a heterogeneous collection of linear molecules, many of which are capable of autonomous replication. Because of the size heterogeneity, a more precise analysis of the termini of this class of DNA was not attempted.

We also obtained transformants after injecting pSA14SB DNA that had been linearized by cleavage with Sall. Transformant DNA was cleaved with HindIII, electrophoresed, blotted, and probed with ${ }^{32} \mathrm{P}$-labeled, nick-translated 


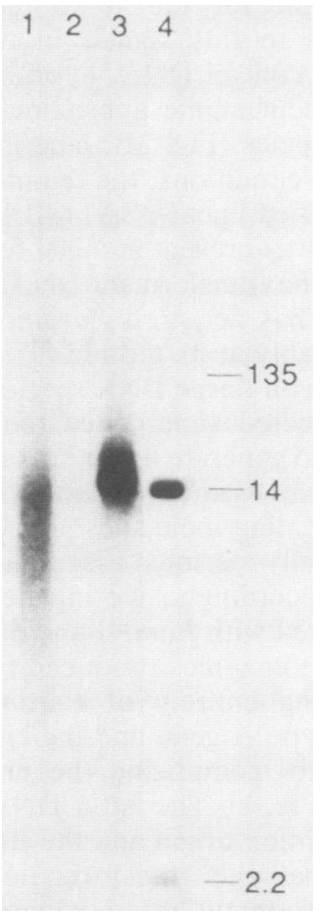

FIG. 4. FIGE analysis of DNA from transformants obtained after injection with Sall-BamHI-cleaved pSA14SB DNA. Lysate DNA was electrophoresed as described in Materials and Methods, transferred to nitrocellulose, and probed with ${ }^{32} \mathrm{P}$-labeled pSA14SB DNA. Lane 1, Transformant lysate from cells injected with linear pSA14SB DNA; lane 2, uninjected cell lysate; lane 3, lysate from cells injected with supercoiled pSA14SB DNA; lane 4, purified pSA14SB DNA cleaved with SalI-BamHI, which generates two fragments of 14 and $2.2 \mathrm{~kb}$. Numbers on the right are size markers in kilobases. The faint band above the $135-\mathrm{kb}$ marker represents hybridization to macronuclear chromosome DNA. The autoradiogram was exposed for $2 \mathrm{~h}$.

pSA14SB DNA. Each of the eight HindIII fragments (Fig. 1) was apparent in transformed DNA and comigrated with its counterpart from purified pSA14SB DNA, except for the two fragments corresponding to the termini. The $3.6-\mathrm{kb}$ fragment from one end was missing and was replaced with a 3.9-kb fragment, and the 2.0-kb fragment from the other end was absent, but hybridization at about $2.3 \mathrm{~kb}$ was apparent (data not shown). We interpret these results to indicate that about 300 base pairs of additional sequences, presumably telomeres, was added to the termini of the injected linear DNA (see below).

Fate of the procaryotic vector fragment after microinjection. Cells injected with BamHI-Sall-cleaved pSA14SB DNA received the $2.2-\mathrm{kb}$ vector fragment, which consists entirely of procaryotic DNA. To examine the fate of this fragment, transformant DNA was electrophoresed, blotted, and probed with labeled pT7/T3-18 DNA. The results (Fig. 6, lane 4) indicate a strong, well-defined hybridization signal at a mobility of about $2.5 \mathrm{~kg}$, about 300 base pairs larger than the input linear fragment. This signal was present only in transformants injected with BamHI-SalI-cleaved pSA14SB DNA and was not detected in transformants injected with supercoiled DNA (Fig. 6, lanes 2 and 3).

Quantitation of the copy number of the $2.5-\mathrm{kb}$ species was carried out by slot-blot DNA hybridization and found to be variable in different lines. Of six lines examined, five had

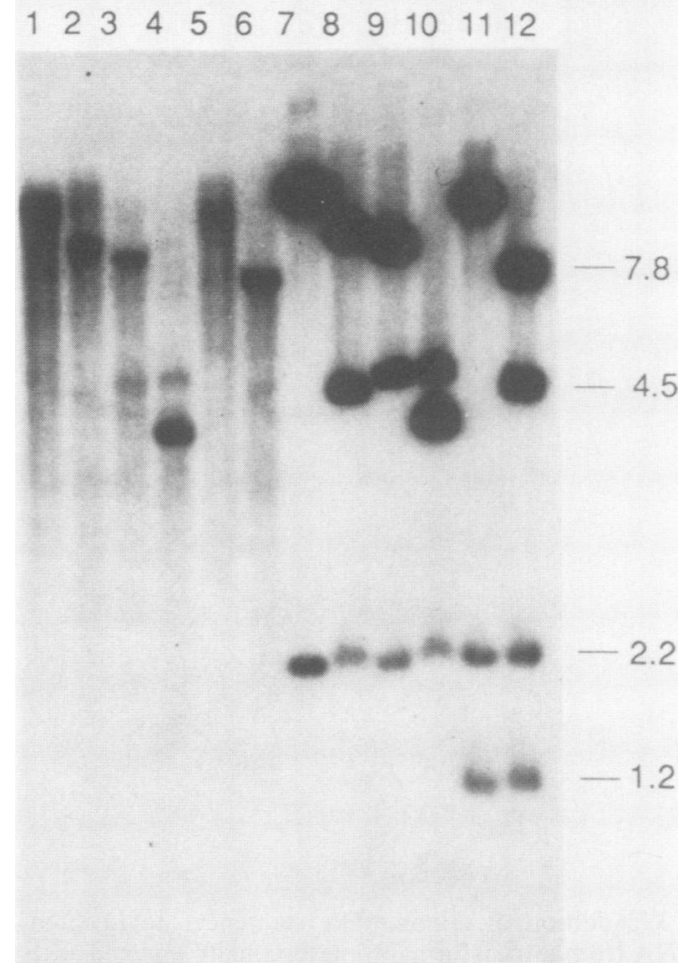

FIG. 5. Restriction enzyme analysis of DNA from transformants derived from cells injected with Sall-BamHI-cleaved pSA14SB DNA. DNA was electrophoresed on a $1 \%$ agarose gel, blotted to nitrocellulose, and probed with ${ }^{32} \mathrm{P}$-labeled pSA14SB DNA. Lanes 1 through 6, DNA from transformants; lanes 7 through 12, purified pSA14SB DNA initially cleaved with Sall-BamHI. Lanes 1 and 7 , No further enzyme cleavage; lanes 2 and 8, cleavage with BglII; lanes 3 and 9, cleavage with EcoRI; lanes 4 and 10, cleavage with EcoRI and BglII; lanes 5 and 11, cleavage with $X b a I$; lanes 6 and 12, cleavage with $X b a I$ and $B g l I I$. The numbers on the right are size markers in kilobases. The expected fragment sizes in kilobases in lanes 7 through 12 are as follows: lane 7,14 and 2.2 ; lane 8, 9.0, 4.5, and 2.2; lane 9, 8.1, 4.9, 2.2, and 0.5 (not shown); lane 10, 4.9, 4.1, 4.0, 2.2, and 0.5 (not shown); lane $11,11.8,2.2$, and 1.2; lane 12, 7.8, $4.5,2.2$, and 1.2. The autoradiogram was exposed for $25 \mathrm{~h}$.

about 10,000 copies per macronucleus; one line had about 100,000 copies (data not shown).

To determine whether the $2.5-\mathrm{kb}$ species was present in a linear form, total transformant DNA was cleaved with ScaI, which cleaves asymmetrically in the linear input vector DNA (see Fig. 9). We expected two distinct bands after $S c a$ I cleavage, representing the two fragments of the vector. Two fragments are present in ScaI-cut DNA, and both migrate with slightly lower mobility than the cognate bands from purified linear T7/T3-18 DNA (Fig. 7, cf. lanes 5 and 6). Also, it is apparent that the bands derived from transformant DNA are somewhat smeared and heterogeneous in size compared with their counterparts from purified DNA. These results strongly suggest that the injected linear procaryotic DNA replicates as a linear form in $\boldsymbol{P}$. tetraurelia and that both termini are modified by addition of sequences after injection. We attribute the smeared appearance of the bands to variable lengths of telomeric sequences added to different termini.

Analysis of the termini of the vector fragment. To examine the possibility that telomerelike sequences were added to the 


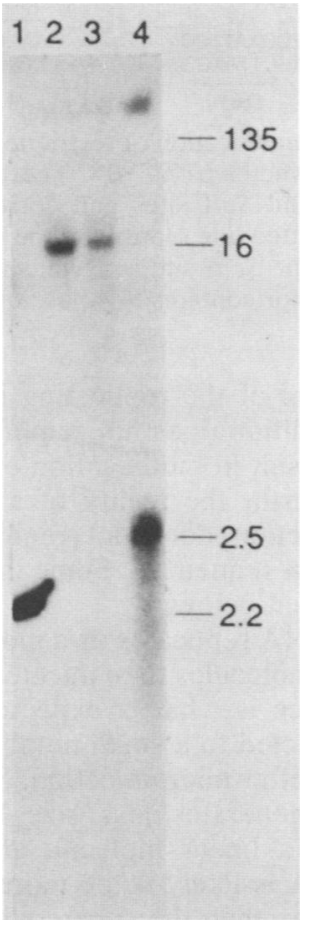

FIG. 6. Detection of microinjected vector sequences in transformants. DNA was electrophoresed on a $1 \%$ agarose gel by the FIGE procedure, transferred to nitrocellulose, and probed with ${ }^{32} \mathrm{P}$-labeled pT7/T3-18 DNA. Lane 1, Purified pSA14SB DNA cleaved with SalI-BamHI; lanes 2 and 3, duplicate samples of transformant DNA derived from cells injected with supercoiled pSA14SB DNA; lane 4, transformant DNA derived from cells injected with SallBamHI-cut pSA14SB DNA. The autoradiogram was exposed for 3 $h$. The numbers on the right refer to the mobility of size markers.

termini of the injected vector DNA, transformant DNA was separated by FIGE, blotted, and probed with the labeled oligonucleotide described earlier. The results (Fig. 8) show that the probe hybridizes to a DNA species of about $2.5 \mathrm{~kb}$. These results demonstrate that nonparamecium DNA injected into the macronucleus is modified by the addition of paramecium telomerelike sequences and is capable of highlevel replication, apparently as unit-length molecules.

The DNA sequence of the injected 2.2-kb molecule permitted a high-resolution analysis of the sequence requirements for telomeric addition in $P$. tetraurelia without subcloning and sequencing of the junction region. The BamHI and $S a l I$ sites are embedded in a polylinker region of the vector; consequently, both ends are flanked with unique restriction sites. By examining whether adjacent restriction sites were still present in the 2.5 -kb species, we were able to draw conclusions about the nature of the termini to which telomeres had been added. For example, a SmaI site is immediately adjacent to the double-stranded BamHI terminus. The removal of a single base pair from this end during the processing involved in telomere addition would eliminate this site. At the SalI terminus, a PstI site is 1 base pair from the double-stranded end. Other sites are located adjacent to the SmaI and PstI sites, permitting an analysis of possible heterogeneity in telomere addition sites (Fig. 9).

Transformant DNA was cut with $S c a$ I to allow inspection of each terminus separately (Fig. 10A and B, lanes 2). Following $S c a$ I cleavage, DNA was cleaved with a variety of endonucleases for which sites exist adjacent to the termini of

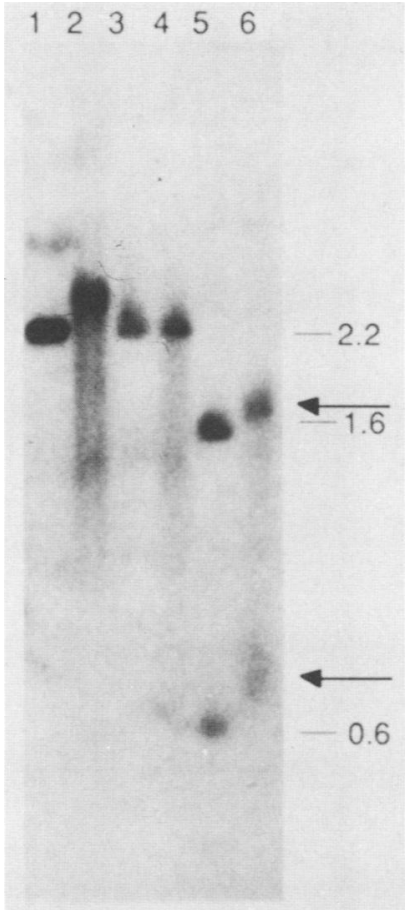

FIG. 7. Restriction enzyme analysis of microinjected vector sequences in transformants. Purified pSA14SB DNA or DNA from transformants microinjected with Sall-BamHI-cut pSA14SB DNA were cleaved with various restriction enzymes, electrophoresed on a $1 \%$ agarose gel, transferred to nitrocellulose, and probed with ${ }^{32}$ P-labeled pT7/T3-18 DNA. Lanes 1, 3, and 5, Purified pSA14SB DNA; lanes 2, 4, and 6, transformant DNA. Lane 1, Cleavage with Sall-BamHI; lane 2, no treatment; lane 3, cleavage with SallBamHI-HindIII-EcoRI; lane 4, cleavage with HindIII-EcoRI; lane 5, cleavage with SalI-BamHI-ScaI; lane 6, cleavage with ScaI. The numbers on the right are size markers in kilobases. The arrows designate the displacement of the hybridizing species in lane 6 relative to that of their counterparts in lane 5 . The autoradiogram was exposed for $16 \mathrm{~h}$.

the injected DNA. The presence of a particular restriction sites could be easily assessed by the conversion of a somewhat smeared band, containing the appended telomerelike sequences, to a sharper band produced by the elimination of these sequences. The cleaved DNA was electrophoresed, blotted, and probed with labeled pT7/T3-18 DNA. The autoradiographic results are shown in Fig. 10A and are presented schematically in Fig. 10B. The closest site to the BamHI end of the linear molecule is a SmaI site, followed by progressively more distant sites for KpnI, SstI, and EcoRI. Sites are retained for each of these enzymes in transformed DNA (Fig. 10, compare lane 2 with lanes 5 through 8). Cleavage at the innermost site, EcoRI, is apparently more complete than at the outermost site, SmaI. This difference could reflect either differential cleavage efficiencies in these particular samples or a slight heterogeneity in the site of telomere addition among the different linear molecules in the population. However, the integrity of the SmaI site in the majority of molecules shows that telomeres can be added without net removal of a single double-stranded base pair from the injected molecule.

A similar analysis of the other end of the linear input molecule revealed that the majority of injected molecules retained the PstI site (Fig. 10A and B, compare lane 2 with lane 12), which required retention of sequences extending to 


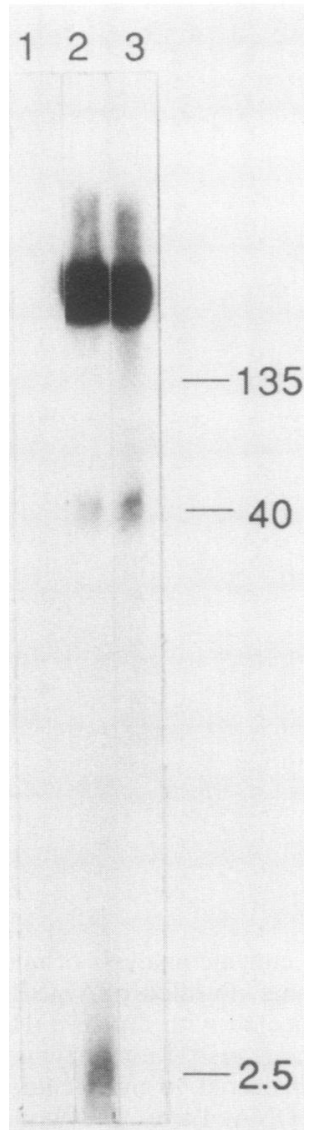

FIG. 8. Analysis of addition of telomerelike sequences to microinjected vector DNA. DNA samples were electrophoresed by the FIGE procedure, transferred to nitrocellulose, and probed with a ${ }^{32} \mathrm{P}$-labeled synthetic oligonucleotide $\left(\mathrm{C}_{4} \mathrm{~A}_{4}\right)_{2}\left(\mathrm{C}_{3} \mathrm{~A}_{3}\right)_{2}$. Lane $1, \mathrm{pS}$ A14SB DNA (500 ng) linearized by cleavage with Sall; lane 2, DNA $(1 \mu \mathrm{g})$ from a transformant line derived from a cell microinjected with SalI-BamHI-cut pSA14SB DNA; lane 3, DNA $(1 \mu \mathrm{g})$ from a noninjected line. The intense hybridization signal in lanes 2 and 3 near the top corresponds to the mobility of bulk macronuclear DNA Note the lack of hybridization in lane 1 at the expected position of mobility of linear pSA14SB DNA. The integrity of pSA14SB DNA in lane 1 was confirmed by its appearance at the expected position after ethidium bromide staining of the gel (not shown). The numbers on the right are size markers in kilobases. The autoradiogram was exposed for $72 \mathrm{~h}$.

1 base pair of the SalI site. As expected, cleavage with either SphI or HindIII released the telomeres from this end (Fig. $10 \mathrm{~A}$ and $\mathrm{B}$, lanes 10 and 11). These results indicate that telomere addition at the SalI terminus also occurs without elimination of input sequences.

In summary, these results show that telomerelike sequences of heterogeneous length are added to the termini of linear microinjected DNA without a requirement for preexisting telomere sequences.

\section{DISCUSSION}

We report on four major features of the transformation system of the ciliated protozoan $P$. tetraurelia. First, injected DNA replicates in an autonomous manner as linear molecules in the macronucleus regardless of whether the DNA injected is initially supercoiled or linear. Second, sequences characteristic of Paramecium telomeres are

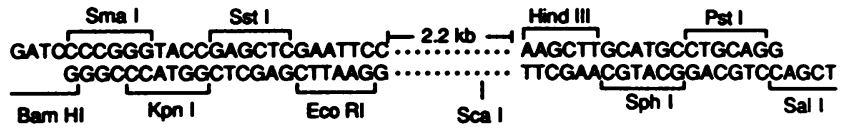

FIG. 9. Sequence and location of restriction cleavage sites at the termini of the 2.2-kb vector pT7/T3-18. The DNA shown is linearized at the BamHI and SalI sites and corresponds to the DNA microinjected. The dotted line represents the majority of the vector sequence. Note that the $S c a I$ site is asymmetrically located in this region. The linear vector contains 5' 4-base overhangs at each end.

added to the termini of the replicating injected molecules. Third, telomere addition does not require specific preexisting sequences or result in modification of preexisting terminal sequences. Fourth, the ability of a DNA molecule to replicate after injection does not require that it initially contain Paramecium sequences. Some implications of these findings are described below.

Injected linear DNA replicates as a more heterogeneously sized collection of molecules than injected supercoiled DNA does. This difference is what is expected if both types of molecules are subjected to a small number of cleavages per molecule during or after microinjection. Single cleavage of a circular molecule generates less size heterogeneity than single cleavage of a linear molecule does. Although the injected linear DNA is likely to be more sensitive to shear during microinjection than the supercoiled DNA is, control experiments indicate that the extent of shear during microinjection is insufficient to generate the distribution seen. Thus, our working hypothesis is that most of the heterogeneity in size is a consequence of cleavages occurring after introduction of the DNA into the macronucleus, during either the establishment or the maintenance phase of transformation.

The pattern of restriction fragments shown in Fig. 5 is not consistent with degradation from the termini of the BamHISalI-cut linear plasmid molecule. Discrete bands identical in size to those of the restriction enzyme-cleaved purified plasmid DNA would not be observed if extensive degradation occurred at the termini. Yet such bands are clearly seen in restricted transformant DNA. Thus, our interpretation of these results is that the majority of the $14-\mathrm{kb}$ input linear DNA is randomly broken into a limited number of fragments which receive telomeres and subsequently replicate. The propensity for breakage is size dependent because smaller linear input molecules, such as the $2.2-\mathrm{kb}$ fragment, are not comparably heterogeneous. Analysis of the termini of the injected 2.2-kb vector DNA provides direct evidence for an absence of degradation from these termini.

An unexpected observation reported earlier for injected supercoiled molecules (10) and confirmed here for linear molecules is their apparent replication to high copy number. We are aware of no other system in which introduced foreign DNA behaves in this manner. In $S$. cerevisiae, input linear DNA is highly recombinogenic if it contains homology with chromosomal DNA and does not replicate autonomously unless it carries either yeast or Tetrahymena telomeric sequences $(17,21,22)$. In many mammalian tissue culture systems, introduction of DNA by either calcium phosphate precipitation $(11)$ or microinjection $(3,7)$ leads to the random integration of a large concatenated molecular complex of DNA (18). Cytogenetic and molecular studies on a number of systems have led to the notion that, in general, broken ends of chromosomes are either highly unstable and subject to degradation or highly recombinogenic $(17,22)$. The macronucleus of $P$. tetraurelia contains 1,000 to 2,000 copies 
of each of 200 to 300 different linear DNA molecules ranging in size from about 100 to $500 \mathrm{~kb}(10,20)$. This situation makes it difficult to monitor the fate of a broken chromosome in the macronucleus. Although the $d 12$ deletion strain retains a small segment of the A gene with which the injected plasmid is homologous, we did not observe extensive integration of the injected sequences into preexisting macronuclear chromosomes.

We have shown that both the $14-$ and the 2.2-kb pieces of BamHI-SalI-cleaved pSA14SB DNA require telomerelike sequences after transformation. The input DNA contains 5'-terminal, 4-nucleotide, single-stranded protruding ends. We do not know the fate of these single-stranded ends after injection. Presumably, they are repaired or trimmed by

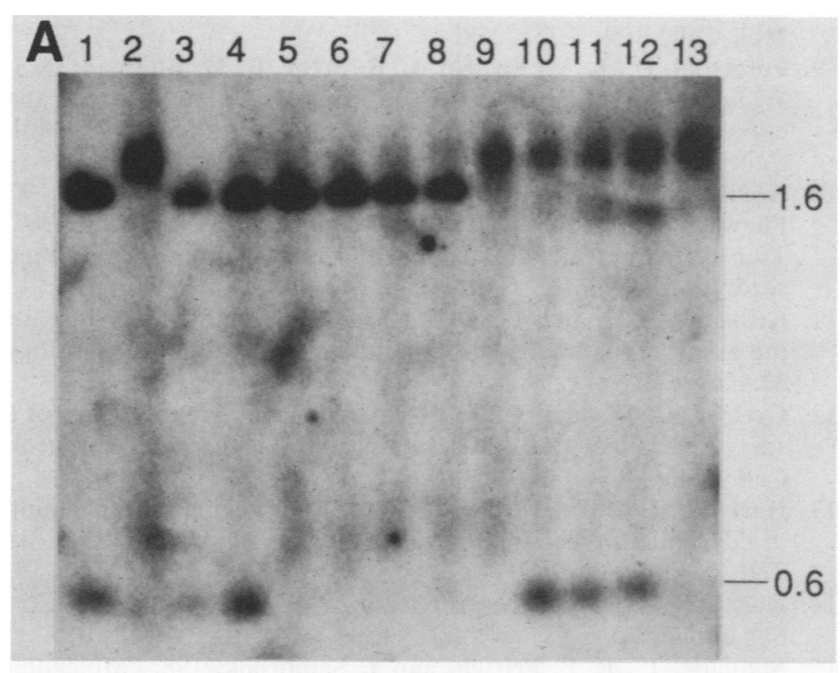

B
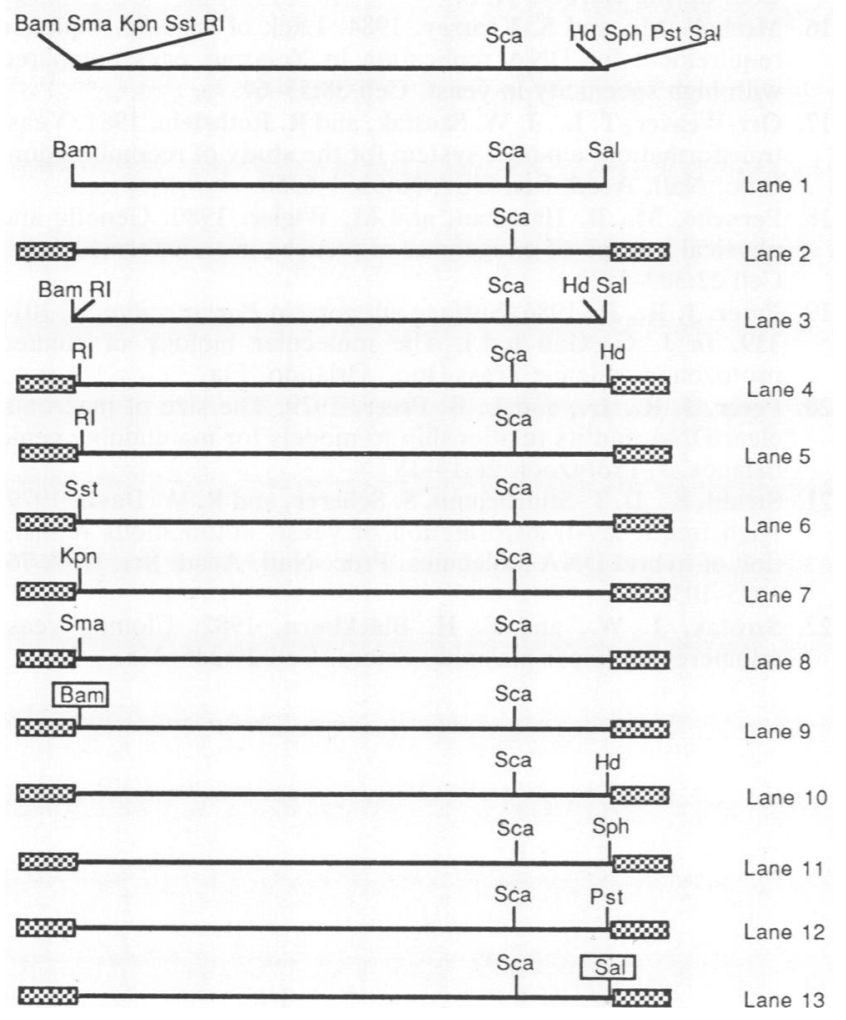

enzymes in the macronucleus. Whatever the fate of the single-stranded termini, it is likely that the double-stranded sequences immediately adjacent to them are completely intact in the replicating molecules. Since neither the singlestranded sequences nor the adjacent double-stranded sequences resemble Paramecium telomeres, our results indicate that the telomere addition system can add telomerelike sequences de novo to heterologous ends. Experiments with Tetrahymena thermophila and $S$. cerevisiae have shown that, in general, telomeres can be added to preexisting telomeric sequences at the ends of chromosomes (for a review, see reference 1). Greider and Blackburn (12) have described a unique enzymatic activity from $T$. thermophila which can add telomeric blocks in vitro to a single-stranded substrate containing telomeric sequences at its terminus. In vitro, this telomere addition activity shows a strict requirement for a preexisting telomere on the substrate. However, in the formation of macronuclear DNA molecules in $T$. thermophila and in hypotrichs such as Oxytricha nova, the addition of telomeres to the ends of processed micronuclear DNA clearly does not require the presence of preexisting telomere sequences $(1,4)$. Thus, the addition of telomeric sequences to nontelomeric ends is a normal part of macronuclear DNA processing. It is also noteworthy that the addition of telomeres during macronuclear DNA formation in $T$. thermophila and in hypotrichs is temporally associated with the extensive DNA replication involved in raising their copy number to the elevated levels characteristic of these systems (1). Thus, the possibility that telomere addition per se confers replication ability must be considered.

Recent work on the $d 48$ mutation of $P$. tetraurelia has shed further light on the limited sequence requirements for telomere addition. The $d 48$ mutant is altered in expression of the type A surface antigen (5). The mutant has a complete A gene in the micronucleus, but its copies in the macronucleus carry a large deletion, suggesting that the $d 48$ mutant is unable to generate correctly the macronuclear chromosome carrying the A gene. Forney and Blackburn (8) have shown that in the $d 48$ mutant and wild-type cells, telomeres are added at a number of different sites. No evidence for a specific recognition sequence was obtained. In the $d 48$ mutant, telomeres were added directly to the truncated A gene at a number of different sites near the $5^{\prime}$ terminus of the gene. These results are consistent with those reported here with injected DNA. Apparently, Paramecium macronuclei

FIG. 10. (A) Analysis of the termini of the vector sequences in a transformant line. Purified vector DNA, pT7/T3-18, or DNA from a transformant line derived from a cell microinjected with SallBamHI-cut pSA14SB DNA was cut with various restriction enzymes, electrophoresed on a $1 \%$ agarose gel, transferred to nitrocellulose, and probed with ${ }^{32}$ P-labeled pT7/T3-18 DNA. Lane 1, Vector cut with ScaI-SalI-BamHI; lane 2, transformant cut with ScaI; lane 3, vector cut with ScaI-SalI-BamHI-HindIII-EcoRI. Lanes 4 through 13, Transformant DNA cut with Scal-HindIIIEcoRI (lane 4), ScaI-EcoRI (lane 5), ScaI-SstI (lane 6), ScaI-KpnI (lane 7), ScaI-SmaI (lane 8), ScaI-BamHI (lane 9), ScaI-HindIII (lane 10), ScaI-SphI (lane 11), ScaI-PstI (lane 12), or ScaI-SalI (lane 13). The autoradiogram was exposed for $24 \mathrm{~h}$. (B) Schematic diagram of the analysis shown in panel A. The lines represent the linearized vector $\mathrm{pT} / \mathrm{T} 3-18$. Checkered rectangles represent telomerelike sequences added to the termini. The enzymes used in the digestions are shown for each lane of the autoradiogram in panel A. The boxed sites in lanes 9 and 13 are half-sites in the linear molecules shown; cleavage is not observed with these enzymes. RI, EcoRI; Hd, HindIII. 
retain the enzymatic machinery to add telomeres to any terminus.

A major question of interest in this system concerns sequence requirements for replication in injected DNA in $P$. tetraurelia. We have shown here that a procaryotic DNA can replicate in $\boldsymbol{P}$. tetraurelia. In general, the copy number of the procaryotic DNA was much lower than that of intact pSA14SB or of the 14-kb segment of pSA14SB containing the serotype A gene. In other experiments, we have shown that the eight HindIII fragments of pSA14SB, ranging in size from 0.5 to $3.6 \mathrm{~kb}$, can also replicate autonomously after injection (data not shown). Preliminary evidence indicates that these fragments also receive telomeres after injection. It seems unlikely that each of these fragments contains a paramecium-specific replication origin. The observation of replication of DNA without apparent sequence specificity is reminiscent of similar results when DNA is microinjected into Xenopus oocytes (16). The permissive state of both systems may be a reflection of their distinct and unusual life-styles. Thus, rapid replication of the Xenopus genome is characteristic of the early embryo, and the brief $S$ period is accomplished by greatly increasing the number of initiation points (2), i.e., presumably by reducing or relaxing any sequence-specific recognition events involved in initiation of replication. An important difference between the Xenopus experiments and those reported here is the form of the replicated DNA. In Xenopus, injected supercoiled DNA replicates in a supercoiled state, while we detected no supercoiled forms.

As described earlier, the macronucleus of $\boldsymbol{P}$. tetraurelia contains about 500,000 separate DNA molecules. Division of the macronucleus occurs in the absence of any visible mitotic apparatus; no information is available on either the mechanical or the molecular basis of segregation of newly replicated DNA at division or about how copy number is established and maintained. The ability to introduce defined DNA into $P$. tetraurelia and monitor its replication and segregation may allow some insight into these problems.

\section{ACKNOWLEDGMENTS}

We thank Louise Preer, Bertina Rudman, and Tim Fitzwater for advice and assistance and Peter Cherbas and Tom Blumenthal for comments on the manuscript.

This work was supported by Public Health Service grants GM34681, GM31745, and GM24212 from the National Institutes of Health to K.J.A., J.R.P., and B.P., respectively. D.G. was supported by a Public Health Service predoctoral training grant from the National Institutes of Health awarded to the Program in Molecular, Cellular and Developmental Biology.

\section{LITERATURE CITED}

1. Blackburn, E. H., and K. M. Karrer. 1986. Genomic reorganization in ciliated protozoans. Annu. Rev. Genet. 20:501-521.

2. Callan, H. G. 1973. DNA replication in the chromosomes of eukaryotes. Cold Spring Harbor Symp. Quant. Biol. 47:11651173.

3. Capecchi, M. R. 1980. High efficiency transformation by direct microinjection of DNA into cultured mammalian cells. Cell 22: 479-488.

4. Dawson, D., and G. Herrick. 1984. Rare internal $C_{4} A_{4}$ repeats in the micronuclear genome of Oxytricha fallax. Mol. Cell. Biol. 4: 2661-2667.

5. Epstein, L. M., and J. D. Forney. 1984. Mendelian and nonMendelian mutations affecting surface antigen expression in Paramecium tetraurelia. Mol. Cell. Biol. 4:1583-1590.

6. Findly, R. C., and J. G. Gall. 1978. Free ribosomal RNA genes in Paramecium are tandemly repeated. Proc. Natl. Acad. Sci. USA 75:3312-3316.

7. Folger, K. R., E. A. Wong, G. Wahl, and M. R. Capecchi. 1982. Patterns of integration of DNA microinjected into cultured mammalian cells: evidence for homologous recombination between injected plasmid DNA molecules. Mol. Cell. Biol. 2: 1372-1387.

8. Forney, J. D., and E. H. Blackburn. 1988. Developmentally controlled telomere addition in wild-type and mutant paramecia. Mol. Cell. Biol. 8:251-258.

9. Forney, J. D., L. M. Epstein, L. B. Preer, B. M. Rudman, D. J. Widmeyer, W. H. Klein, and J. R. Preer, Jr. 1983. Structure and expression of genes for surface proteins in Paramecium. Mol. Cell. Biol. 3:466-474.

10. Godiska, R., K. J. Aufderheide, D. Gilley, P. Hendrie, T. Fitzwater, L. B. Preer, B. Polisky, and J. R. Preer, Jr. 1987. Transformation of Paramecium by microinjection of a cloned serotype gene. Proc. Natl. Acad. Sci. USA 84:7590-7594.

11. Graham, F. L., and A. van der Eb. 1973. A new technique for the assay of infectivity of human adenovirus 5 DNA. Virology 52:456-467.

12. Greider, C. W., and E. H. Blackburn. 1985. Identification of a specific terminal transferase activity in Tetrahymena extracts. Cell 43:405-413.

13. Harland, R. M., and R. A. Laskey. 1980. Regulated replication of DNA microinjected into eggs of Xenopus laevis. Cell 21:761771.

14. Kornberg, A. 1980. DNA replication. W. H. Freeman and Co., San Francisco.

15. Maniatis, T., E. F. Fritsch, and J. Sambrook. 1982. Molecular cloning: a laboratory manual. Cold Spring Harbor Laboratory, Cold Spring Harbor, N.Y.

16. Mechali, M., and S. Kearsey. 1984. Lack of specific sequence requirement for DNA replication in Xenopus eggs compared with high specificity in yeast. Cell 38:55-64.

17. Orr-Weaver, T. L., J. W. Szostak, and R. Rothstein. 1981. Yeast transformation: a model system for the study of recombination. Proc. Natl. Acad. Sci. USA 78:6354-6358.

18. Perucho, M., D. Hanahan, and M. Wigler. 1980. Genetic and physical linkage of exogenous sequences in transformed cells. Cell 22:309-317.

19. Preer, J. R., Jr. 1986. Surface antigens in Paramecium, p. $301-$ 339. In J. G. Gall (ed.), The molecular biology of ciliated protozoa. Academic Press, Inc., Orlando, Fla.

20. Preer, J. R., Jr., and L. B. Preer. 1979. The size of macronuclear DNA and its relationship to models for maintaining genic balance. J. Protozool. 26:14-18.

21. Struhl, K., D. T. Stinchcomb, S. Scherer, and R. W. Davis. 1979. High frequency transformation of yeast: autonomous replication of hybrid DNA molecules. Proc. Natl. Acad. Sci. USA 76: 1035-1039.

22. Szostak, J. W., and E. H. Blackburn. 1982. Cloning yeast telomeres on linear plasmid vectors. Cell 29:245-255. 